\title{
Size dependence, coherence, and scaling in turbulent coupled-map lattices
}

\author{
Bohr, Tomas; Christensen, Ole Bøssing
}

Published in:

Physical Review Letters

Link to article, DOI:

10.1103/PhysRevLett.63.2161

Publication date:

1989

Document Version

Publisher's PDF, also known as Version of record

Link back to DTU Orbit

Citation (APA):

Bohr, T., \& Christensen, O. B. (1989). Size dependence, coherence, and scaling in turbulent coupled-map lattices. Physical Review Letters, 63(20), 2161-2164. https://doi.org/10.1103/PhysRevLett.63.2161

\section{General rights}

Copyright and moral rights for the publications made accessible in the public portal are retained by the authors and/or other copyright owners and it is a condition of accessing publications that users recognise and abide by the legal requirements associated with these rights.

- Users may download and print one copy of any publication from the public portal for the purpose of private study or research.

- You may not further distribute the material or use it for any profit-making activity or commercial gain

- You may freely distribute the URL identifying the publication in the public portal 


\title{
PHYSICAL REVIEW LETTERS
}

\begin{tabular}{lcc}
\hline Volume 63 & 13 NOVEMBER 1989 & NuMBER 20 \\
\hline \hline
\end{tabular}

Size Dependence, Coherence, and Scaling in Turbulent Coupled-Map Lattices

\author{
Tomas Bohr \\ The Niels Bohr Institute, Blegdamsvej 17, 2100 Copenhagen, Denmark \\ Ole Bøssing Christensen \\ The Niels Bohr Institute, Blegdamsvej 17, 2100 Copenhagen, Denmark \\ and Laboratory of Applied Physics, The Technical University of Denmark, \\ 2800 Lyngby, Denmark \\ (Received 27 March 1989)
}

\begin{abstract}
In this paper the crossover from "small" to "large" chaotic systems is studied. The behavior of the largest Lyapunov exponent in a system of coupled chaotic maps shows that this crossover is remarkably sharp, and allows us to define a coherence length beyond which the system is effectively large. Between the coherent chaos of the small system and the incoherent chaos (turbulence) of the large one there is a stable window starting at the linear instability point for the uniform chaotic state in which the lattice becomes effectively one dimensional. The scaling of the coherence length close to the onset of turbulence is investigated and compared to recent predictions.
\end{abstract}

PACS numbers: $05.45 .+\mathrm{b}, 47.25 .-\mathrm{c}, 64.60 . \mathrm{Fr}$

The study of coupled-map lattices ${ }^{1,2}$ has become a very fruitful way of building up intuition and knowledge about extended dynamical systems-like hydrodynamical turbulence or inhomogeneous chemical reactions - and testing and generalizing the methods used to describe low-dimensional chaotic systems in a context where spatial degrees of freedom are important. Chaotic motion in extended systems is typically incoherent or "high dimensional." ${ }_{3}$ If the system is large, enough different parts become decorrelated and the dimension of the "attractor" will presumably grow in proportion to the volume of the system. Phrased differently: An extended dynamical system is characterized by a finite coherence (or correlation) length ${ }^{3}$ such that points separated by distances much larger than that are completely decorrelated.

The coherence length is thus a very important quantity, whose magnitude, relative to the system size, determines whether the system is "large" or "small"; and it is of crucial importance to understand its dependence on parameters and its relation to other characteristic lengths in the system. The correlation length is usually defined via the exponential decay of an equal time correlation function (two-point function). It is, in general, very hard numerically to obtain a nice exponential decay in coupled-map systems. In one dimension one can handle very large systems, but they "equilibrate" very slowly due to the presence of almost frozen defects; ${ }^{1}$ in two (or higher) dimensions the equilibration properties are much better, ${ }^{3}$ but the size limitations become severe. For a special class of one-dimensional coupled maps (linear discontinuous) ${ }^{4}$ one has been able to see reasonable exponential decays in the correlation function. A more practical method has been suggested recently ${ }^{5}$ in which one computes the decay rate for the exponentially decreasing spatial power spectrum, although the physical meaning of the characteristic length scale determined in this way is not always clear.

In the following we shall look instead at systems of different sizes to see how the characteristics of the dynamics change. In low-dimensional chaotic systems a very useful quantity is the (largest) Lyapunov exponent which characterizes the rate of exponential separation of points in phase space that are initially very close. This concept can be applied to extended dynamical systems like partial differential equations ${ }^{6}$ or coupled-map lattices, ${ }^{7}$ but very little is known about the dependence of this quantity (or related quantities) on the size of the 
system, i.e., the crossover from small to large. We shall see that this dependence allows us to pick out important characteristic lengths related to the coherence of the system.

We consider a standard diffusively coupled-map lattice: ${ }^{1}$ a two-dimensional cubic lattice indexed by $(i, j)$ where $i, j=1,2, \ldots, L$. On each site we have a scalar field $u_{n}(i, j)$, where $n$ is the discrete time, and we always assume periodic boundary conditions in $i$ and $j$. The dynamics are given by

$$
\begin{aligned}
u_{n+1}(i, j)= & (1-\epsilon) f\left(u_{n}(i, j)\right) \\
& +\frac{1}{4} \epsilon \sum_{\mathrm{nn} i^{\prime}, j^{\prime}} f\left(u_{n}\left(i^{\prime}, j^{\prime}\right)\right),
\end{aligned}
$$

where $\left(i^{\prime}, j^{\prime}\right)$ are nearest neighbors of $(i, j)$. The function $f(x)$ is taken to be some nonlinear map that can sustain chaotic motion, e.g., the logistic map $f(x)$ $=R x(1-x)$. The logistic map has a period-doubling cascade $^{8}$ with accumulation point $R_{c}=3.5699456 \ldots$ and for $R_{c}<R<4$ the attractor is either chaotic or periodic, but even in the periodic windows the periodic attractor coexists with a chaotic repellor giving rise to chaotic transients. For the coupled system, transients increase rapidly with the system size $L$ and even for moderate sizes the periodic windows are never seen with noisy initial conditions. ${ }^{3,9}$

Chaotic motion is characterized by a positive Lyapunov exponent defined by ${ }^{10}$

$$
\lambda=\lim _{N \rightarrow \infty} \frac{1}{N} \sum_{i=0}^{N-1} \ln \left|f^{\prime}\left(x_{i}\right)\right|,
$$

where $x_{i}=f\left(x_{i-1}\right)$ and $x_{0}$ is the starting point of which $\lambda$ is independent. For the coupled system (1) one can similarly define Lyapunov exponents by looking at the variation

$$
\begin{aligned}
d u_{n+1}(i, j)= & (1-\epsilon) f^{\prime}\left(u_{n}(i, j)\right) d u_{n}(i, j) \\
& +\frac{1}{4} \epsilon \sum_{\mathrm{nn}} f^{\prime}\left(u_{n}\left(i^{\prime}, j^{\prime}\right)\right) d u_{n}\left(i^{\prime}, j^{\prime}\right)
\end{aligned}
$$

For a lattice of size $L$ one can, in principle, define $L^{2}$ Lyapunov exponents, such that the sum of the first $i$ characterizes the exponential growth or decay of an $i$ dimensional hypervolume in phase space. Here we shall mostly confine our attention to the largest one which describes the growth of distances analogously to the single-map case. Numerically this (as well as the lower exponents) can be found by standard techniques. ${ }^{6,7,10}$

Such calculations were done on the system (1) in Ref. 11 and it was noted that the value of $\lambda$ for large lattices is roughly proportional to that of the single map $\lambda_{0}$ (around a factor of 2 smaller) and specifically scales with the same exponent as $R \rightarrow R_{c}^{+}$. For a very small lattice we expect, however, to find a value close to $\lambda_{0}$, and thus we might ask how the crossover takes place. Does it show the characteristic length scales of the system?
A specific example is shown in Fig. 1, where the largest Lyapunov exponent of the map (1) with $R=3.5732$. . i.e., $\ln \left[\left(R-R_{c}\right) / R_{c}\right]=-7$ \} and $\epsilon=0.4$ is plotted against the lattice size $L$, which varies from 1 to 50. For each lattice, noisy initial conditions were used. The first 5000 iterates were discarded and the next $N=10000$ iterates were used to evaluate $\lambda$. The uncertainty was estimated by comparing to the value of $\lambda$ obtained halfway (i.e., with $N=5000$ ) - it is of the order of a few times the dot size in the figure.

For small lattices ( $L \leq 8$ in the specific example) the Lyapunov exponent is close (within $5 \%$ ) to the singlemap value 0.059 . Between $L=8$ and $L=9$ it drops to a value close to zero where it remains up to $L=13$. Between $L=13$ and 15 it rises to its "large-system" value; all the way up to $L=50$ it remains close (again within $5 \%)$ to $\lambda_{\infty}=0.030$. A closer look at the motion reveals that the lattice becomes absolutely flat for $L \leq 8$; i.e., the chaotic motion of each map is completely in phase, although the initial conditions were noisy (the differences in $\lambda$ are numerical inaccuracy due to the finite waiting times).

Between $L=9$ and $L=12$ the state is modulated in one direction and flat in the other. It is easy to see that this transition is simply determined by linear stability of the uniform chaotic state. In the chaotic state very long waves are unstable. A perturbation $\delta_{0} e^{i q \cdot \mathbf{x}}$ of the flat state will grow as $e^{\lambda_{\epsilon}(q) n}$, where ${ }^{4,12}$

$$
\begin{aligned}
\lambda_{\epsilon}(\mathbf{q}) & =\lambda_{0}+\ln \left|1-\epsilon\left[1-\frac{1}{2}\left(\cos q_{1}+\cos q_{2}\right)\right]\right| \\
& \approx \lambda_{0}-\left(\frac{1}{2} q\right)^{2} \epsilon .
\end{aligned}
$$

Here $\lambda_{0}$ is the Lyapunov exponent of the single map $f$ and the approximate expression is valid for small $q$. The allowed wave vectors are of the form $q=(2 \pi / L)\left(n_{1}, n_{2}\right)$, where $n_{1}$ and $n_{2}$ are integers. Thus the first wave vector

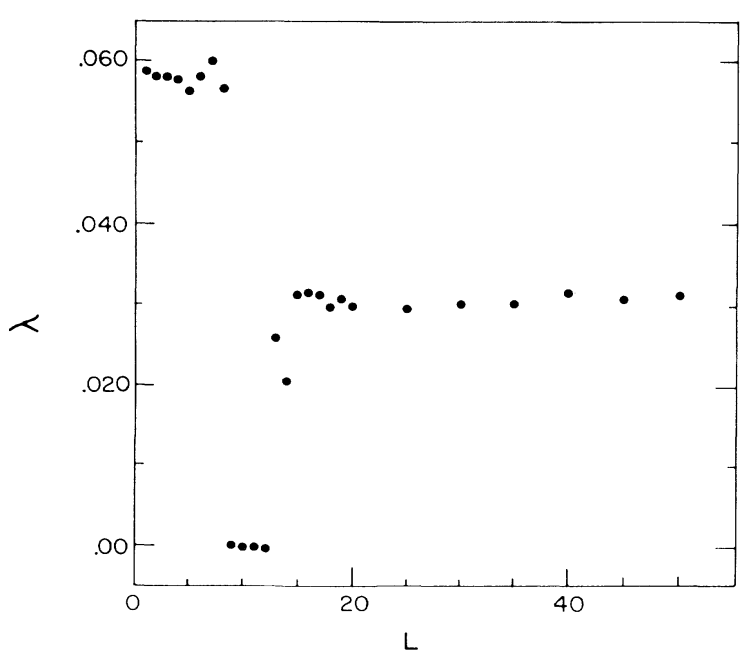

FIG. 1. Size dependence of the largest Lyapunov exponent. $\ln \left[\left(R-R_{c}\right) / R_{c}\right]=-7.0$ and $\epsilon=0.4$. 
$\mathbf{q}=(2 \pi / L)(1,0)$ or $\mathbf{q}=(2 \pi / L)(0,1)$ becomes unstable at system size $L_{1}$ determined by $\lambda_{\epsilon}\left[q=\left(2 \pi / L_{1}, 0\right)\right]=0$ which for large $L_{1}$ means $L_{1} \approx \pi\left(\epsilon / \lambda_{0}\right)^{1 / 2}$.

For the parameters corresponding to Fig. 1 we get $L_{1}=8.02$ which means that $L=9$ is the first unstable system. Strangely enough at first sight the system responds to this instability by becoming stable- the vanishing Lyapunov exponent indicates nonchaotic, almost periodic behavior. On further reflection this is perhaps not so strange. Above $L_{1}$ the system can suddenly use the spatial degrees of freedom (at least in one direction) and thus it has much more phase space to search for stable motion and can remain coherent by lowering the Lyapunov exponent. Since the lattice is effectively one dimensional in this range, these states are analogous to the "pattern selection" found in Ref. 13.

At $L=13$ modulations in both directions appear. This happens roughly at $L=\sqrt{2} L_{1}$ corresponding to $\lambda_{\epsilon}[q=(2 \pi / L)(1,1)]=0$, i.e., the instability of the second mode in the uniform state. Now the system begins to have problems keeping its different parts together and for increasing $L$ the dynamics quickly approaches largesystem behavior with a new Lyapunov exponent reflecting the fact that different parts are dephased. Already at $L=L_{c}=15$ the value of $\lambda$ is indistinguishable from its value at $L=50$ indicating that the incoherent averaging responsible for its large-system value is now effective. The lowering of the Lyapunov exponent com-

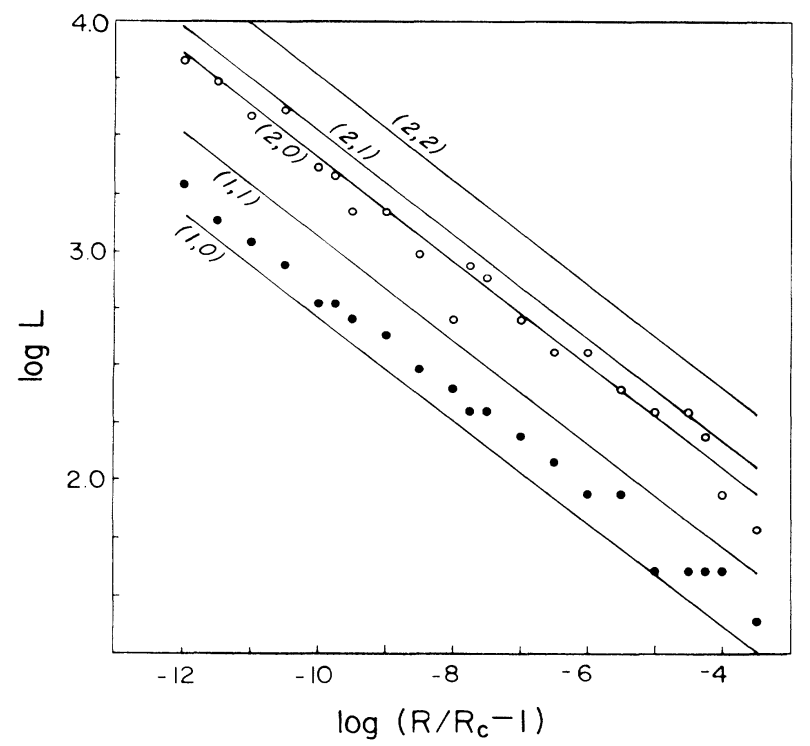

FIG. 2. Scaling of $L_{1}$ (filled circles) and $L_{c}$ (empty circles). Along the $x$ axis is $\ln \left[\left(R-R_{c}\right) / R_{c}\right]$ and $\epsilon$ is 0.4 . The straight lines have slope $-\log 2 / 2 \log \delta \approx-0.2249$ and give the instability thresholds (in the uniform state) for wave vectors $\mathrm{q}=(2 \pi / L)\left(n_{1}, n_{2}\right)$, where $\left(n_{1}, n_{2}\right)$ are indicated on each line. Here the long-wavelength approximation in (5) is used together with the scaling law (6). The full expression (5) would bend down slightly to the right, but scale in the same way to the left. pared to the single map is caused by the existence of both positive and negative slopes $f(u)$. Continuity along the lattice (enforced by the coupling) implies that between regions with positive slopes and regions with negative ones there must be regions where the displacement is very small thus lowering the overall growth rate.

For other values of the parameter $R$ we have found similar behavior. There is a size $L_{1}$ at which the Lyapunov exponent jumps down to a value close to zero. At a later value $L_{2}, \lambda$ starts moving up again and at $L_{c}$ its value is indistinguishable from that of the infinite system (extrapolating from $L \approx 50$ ). In Fig. 2 we have shown $L_{1}$ and $L_{c}$ against $R-R_{c}$ in a $\log -\log$ plot. We have chosen $L_{1}$ as the smallest "stable" size and we have chosen $L_{c}$ as the smallest $L$ for which the difference between $\lambda(L)$ and the "infinite-system" value $\lambda_{\infty}$ is less than, say, $10 \%$. For the highest values of $L_{c}$ (the top left points) transients become very long and our data are somewhat less accurate. ${ }^{14}$ For small $L$ the staircase nature of the plot due to the discreteness of $L$ is evident. The straight lines in Fig. 2 have slope $-v$, where $v=\log 2 / 2 \log \delta=0.2249 \ldots$ ( $\delta$ is Feigenbaum's constant ${ }^{8}$ ) and are (approximately) the linear stability thresholds for the uniform chaotic state for different modes as marked on the lines. They are obtained from (3) using the scaling behavior ${ }^{8} \lambda_{0} \approx a\left(R-R_{c}\right)^{2 v}$ with $a$ estimated from our data. It is seen that, very roughly, $L_{c}$ is a factor of (slightly less than) 2 larger than $L_{1}$ and that they seem to scale in the same way when $R$ approaches $R_{c}$ :

$$
L_{c} \sim L_{1} \sim\left(R-R_{c}\right)^{-v} .
$$

The number of positive Lyapunov exponents is a rough measure of the dimension (lower bound if the measure is sufficiently smooth ${ }^{10}$ ). This number changes rapidly around $L_{c}$. For the parameters of Fig. 1 it is zero between $L_{1}=9$ and $L=12$. From 3 at $L=13$ it changes to 6 at $L_{c}=15$ so indeed the system is quickly getting high dimensional. Figure 3 shows the number of positive ex-

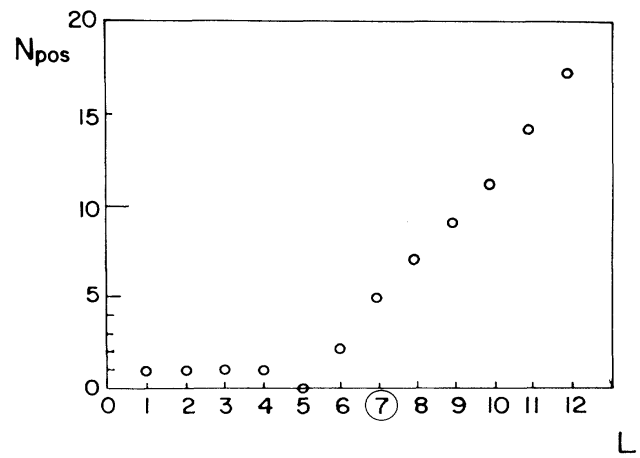

FIG. 3. The number of positive Lyapunov exponents as a function of size for $R=3.6353\left\{\ln \left[\left(R-R_{c}\right) / R_{c}\right]=-4.0\right\}$ and $\epsilon=0.4$. Here $L_{c}=7$ as shown by the circle on the $x$ axis. 
ponents as function of system size for a larger $R$ (where the relevant $L$ values are smaller and computations easier). Here $L_{c}=7$ and we see a persistent growth in the number of active modes. ${ }^{15}$ This means that we can view $L_{c}$ as a coherence length: Systems larger than $L_{c}$ are incoherent, consisting of several independent parts, and thus effectively large. Naively one might have taken $L_{1}$ as a coherence length, but that would be misleading. At $L_{1}$ the uniform state is lost, but coherence is still maintained.

The scaling relation (4) is in agreement with numerical computations ${ }^{4}$ of the correlation length in a onedimensional chain of linear discontinuous maps of the form $f(x)=r x(\bmod 1)$. It also agrees with the scaling of the decay rate of the exponential spatial power spectrum $^{5}$ (although this quantity shows nonuniversal behavior further away from criticality). It disagrees, however, with numerical results ${ }^{11}$ for the two-dimensional coupled-map system (1) obtained by interpreting the first zero of the correlation function as a measure of the correlation length. Our later analysis ${ }^{5}$ has shown that it is very difficult numerically, at least with the sizes of lattices that are available to us, to see an exponentially decreasing correlation function. The first zero is therefore not very useful and can vary strongly due to local structures. Further the interval of $R$ values studied in Ref. 11 was very short (due to the rapid rise of the measured length scale $)$, corresponding only to $-6.15<\ln [(R$ $\left.\left.-R_{c}\right) / R_{c}\right]<-4.8$ in Fig. 2.

Some analytic work ${ }^{4,12}$ has been done to support the scaling law (4). They are, however, based on the notion that the state of the system is almost uniform; and thus one is basically calculating $L_{1}$. As seen from our results the state close to $L_{c}$ is in no way close to being uniform so a different approach seems to be required. One can obtain an upper bound to the coherence length based on the existence of a finite maximal speed, $c$, by which disturbances can spread. Thus the coherence length $\xi$ and the largest Lyapunov exponent $\lambda$ should satisfy ${ }^{3}$ $\xi \leq$ const $\times c \lambda^{-1}$. In models like (1) with only nearestneighbor coupling $c$ cannot exceed unity, but in fact one would expect $c$ to depend on the Lyapunov exponent. ${ }^{16}$ Consider a small local perturbation $d u_{0}(\mathbf{r})$ at $\mathbf{r}=\mathbf{r}_{0}$ and time 0 , and let us ask for the distribution $d u_{n}(\mathbf{r})$ at time $n$. This can be found from (2) which of course, in general, is very hard to solve. If we approximate (2) by setting all $f^{\prime}\left(u_{m}(i, j)\right)=$ const $=e^{\lambda}$ [such as for the chaotic $\operatorname{map} f(x)=r x(\bmod ) 1]$ we find

$$
d u_{n}(\mathbf{r})=d u_{0} e^{\left(\lambda n-\left|\mathbf{r}-\mathbf{r}_{0}\right|^{2} / \epsilon n\right)}
$$

Thus we get exponential amplification in any system of coordinates moving with speed less than $c_{\text {max }}$, where $c_{\max }=(\epsilon \lambda)^{1 / 2}$. This leads to the estimate $\xi \sim \lambda^{-1 / 2}$ which again leads to (4) using the scaling relation for $\lambda$.

We would like to thank G. Grinstein, M. H. Jensen, A. W. Pedersen, H. G. Schuster, and D. Umberger for many helpful and illuminating discussions. O.B.C. would like to thank the Carlsberg Foundation for a scholarship which supported part of this work.

${ }^{1}$ J. P. Crutchfield and K. Kaneko, in Directions in Chaos, edited by Hao Bai-Lin (World Scientific, Singapore, 1987), Vol. 1.

${ }^{2}$ H. Chaté and P. Manneville, Physica (Amsterdam) D 32, 409 (1988).

${ }^{3}$ T. Bohr, G. Grinstein, Yu He, and C. Jayaprakash, Phys. Rev. Lett. 58, 2155 (1987).

${ }^{4}$ F. Kaspar and H. G. Schuster, Phys. Lett. 113A, 451 (1986).

${ }^{5}$ O. B. Christensen, thesis, University of Copenhagen, 1987 (unpublished).

${ }^{6}$ Y. Pomeau, A. Pumir, and P. Pelce, J. Stat. Phys. 37, 39 (1984).

${ }^{7}$ K. Kaneko, Prog. Theor. Phys. 74, 1033 (1985).

${ }^{8}$ Universality in Chaos, edited by P. Cvitanović (Adam Hilger, Bristol, 1984).

${ }^{9}$ K. Kaneko and J. P. Crutchfield, Phys. Rev. Lett. 60, 2715 (1988).

${ }^{10}$ J.-P. Eckmann and D. Ruelle, Rev. Mod. Phys. 67, 617 (1985).

${ }^{11}$ D. R. Rasmussen and T. Bohr, Phys. Lett. A 125, 107 (1987).

${ }^{12}$ S. P. Kuznetsov and A. S. Pikovsky, Physica (Amsterdam) D 19, 384 (1986); A. S. Pikovski, Z. Phys. B 55, 149 (1984).

${ }^{13}$ K. Kaneko, Physica (Amsterdam) D 34, 1 (1989).

${ }^{14}$ One should note that the map (1), as generic nonequilibrium dynamical systems, is "multistable," i.e., many stable states exist for a given set of parameters. Among these are periodic states, for example, the uniform "window" states discussed above. The states appearing in plots like Fig. 1 should be stable against noise, and, if other states appear, one should select the most stable one by looking at the motion of domain walls (Ref. 3). I would like to thank W.v.d. Water for discussions of this issue.

${ }^{15}$ For large $L$ one would expect this number to scale as the system area, analogously to the linear behavior in $L$ for $1 \mathrm{D}$ systems (Ref. 6), but our data are insufficient to verify this.

${ }^{16} \mathrm{We}$ would like to thank P. Grassberger for discussions of this point. 